\title{
Prediction and Diagnosis of Respiratory Disease by Combining Convolutional Neural Network and Bi- directional Long Short-Term Memory Methods
}

\section{Li Li}

Department of Respiratory and Critical Care Medicine, First People's Hospital of Kashi

\section{Alimu Ayiguli}

Department of Clinical Research Center of Infectious Diseases (Pulmonary Tuberculosis), First People's Hospital of Kashi

\section{Qiyun Luan}

Department of Clinical Research Center of Infectious Diseases (Pulmonary Tuberculosis), First People's Hospital of Kashi

\section{Boyi Yang}

Department of Preventive Medicine, School of Public Health, Sun Yatsen University

Hui Gong

Department of Clinical Research Center of Infectious Diseases (Pulmonary Tuberculosis), First People's Hospital of Kashi

\section{Abudureherman Zulipikaer}

Department of Clinical Research Center of Infectious Diseases (Pulmonary Tuberculosis), First People's Hospital of Kashi

\section{Jingran Xu}

Department of Clinical Research Center of Infectious Diseases (Pulmonary Tuberculosis), First People's Hospital of Kashi

\section{Xuemei Zhong}

Department of Respiratory and Critical Care Medicine, First People's Hospital of Kashi

\section{Yilamujiang Subinuer}

Department of Clinical Research Center of Infectious Diseases (Pulmonary Tuberculosis), First People's Hospital of Kashi

\section{Jiangtao Ren}

Department of Software, Sun Yat-Sen University

\section{Xiaoguang Zou ( $\sim$ ZXGKashi@yeah.net)}

Department of Clinical Research Center of Infectious Diseases (Pulmonary Tuberculosis), First People's Hospital of Kashi

\section{Research Article}


Keywords: Respiratory disease, Convolutional neural network, Long-short-term memory network, Predictive diagnosis, Medical records

Posted Date: February 17th, 2022

DOI: https://doi.org/10.21203/rs.3.rs-1332028/v1

License: (c) (1) This work is licensed under a Creative Commons Attribution 4.0 International License. Read Full License 


\section{Prediction and Diagnosis of Respiratory Disease by Combining Convolutional} Neural Network and Bi-directional Long Short-Term Memory Methods

Li Li ${ }^{1,2,5+}$,Alimu Ayiguli ${ }^{2+}$, Qiyun Luan ${ }^{2+}$,Boyi Yang ${ }^{4}$,Hui Gong ${ }^{2}$, Abudureherman

Zulipikaer ${ }^{2}$,Jingran $\mathrm{Xu}^{2}$, Xuemei Zhong ${ }^{1}$,Yilamujiang Subinuer ${ }^{2}$,Jiangtao

$\operatorname{Ren}^{3 *}$,Xiaoguang Zou ${ }^{2 *}$

${ }^{1}$ Department of Respiratory and Critical Care Medicine, First People's Hospital of

Kashi, Kashi, Xinjiang, China

${ }^{2}$ Department of Clinical Research Center of Infectious Diseases (Pulmonary

Tuberculosis), First People's Hospital of Kashi, Kashi, Xinjiang, China

${ }^{3}$ Department of Software, Sun Yat-Sen University, Guangzhou, Guangdong,China

${ }^{4}$ Department of Preventive Medicine, School of Public Health, Sun Yatsen University,

Guangzhou, Guangdong,China

${ }^{5}$ State Key Laboratory of Pathogenesis, Prevention and Treatment of High Incidence

Diseases in Central Asia, Xinjiang Medical University

${ }^{+}$Li Li, Alimu Ayiguli, and Qiyun Luan are contributed equally to this work as primary authors (Co-first author).

*Corresponding author: Xiaoguang Zou, First People's Hospital of Kashi, 120 Yinbin Avenue, Kashi, 844000, China,E-mail: ZXGKashi@yeah.net. Jiangtao Ren,132East Outer Ring Road, Sun Yat-Sen University, Guangzhou, 510000,China,E-mail: issrjt@mail.sysu.edu.cn. 


\section{Abstract}

Objective Based on the respiratory disease big data platform in southern Xinjiang, we established a model that predicted and diagnosed chronic obstructive pulmonary disease, bronchiectasis, pulmonary embolism and pulmonary tuberculosis, and provided assistance for primary physicians. Methods The method combined convolutional neural network (CNN) and long-short-term memory network (LSTM) for prediction and diagnosis of respiratory diseases. We collected the medical records of inpatients in the respiratory department, including: chief complaint, history of present illness, and chest computed tomography. Preprocessing of clinical records with“ jieba" word segmentation module, and the Bidirectional Encoder Representation from Transformers (BERT) model was used to perform word vectorization on the text. The partial and total information of the fused feature set was encoded by convolutional layers, while LSTM layers decoded the encoded information. Results The precisions of traditional machine-learning, deep-learning methods and our proposed method were $0.6,0.81,0.89$, and $F 1$ scores were $0.6,0.81$, 0.88, respectively. Conclusion Compared with traditional machine learning and deep-learning methods that our proposed method had a significantly higher performance, and provided precise identification of respiratory disease.

Key words: Respiratory disease; Convolutional neural network; Long-short-term memory network; Predictive diagnosis; Medical records 


\section{Introduction}

Respiratory diseases, including pulmonary tuberculosis (PTB), chronic obstructive pulmonary disease (COPD), pulmonary thromboembolism (PTE), and bronchiectasis, are among the most common diseases clinically. These diseases have common symptoms such as cough, sputum expectoration, wheezing, and chest pain, but the treatment and follow-up of each disease are completely different [1-4]. The similar symptoms among these diseases make timely diagnosis difficult. Misdiagnosis is common in primary hospitals, and can lead to inappropriate treatment, prolonged recovery time, and potential deterioration, and limited experience of doctors at primary hospitals also worsens the situation [5].

The dry climate, air pollution and rural biofuels have led to a high incidence of chronic airway diseases, and the limited experience and medical equipment of doctors in primary hospitals in Kashi, Xinjiang, China make it difficult to identify similar diseases [6]. Therefore, we established a respiratory system big data platform in Kashi, using machine learning, natural language recognition and extraction methods to discuss the information in patients' electronic medical records, and established algorithm models to achieve high accuracy through model autonomous learning.In practical applications, machine learning methods provide technical support for precision medicine and efficient medicine [7] .

Research shows that machine learning has been applied in medical treatment, including diagnosis, recurrence prediction, and medication [8]. The purpose of machine learning is performing high precision classification or discrimination of 
unknown predicted diseases through autonomous learning and data analysis. In a world of ever-growing data where hospitals are slowly adopting big data systems [9], there are major benefits to using data analytics in the healthcare system to provide insights, augment diagnosis, improve outcomes, and reduce costs [10]. In particular, successful implementation of machine learning enhances the work of medical experts and improves the efficiency of the healthcare system [11]. Significant improvements in diagnostic accuracy have been shown through the performance of machine-learning models along with clinicians [12].

In this study, we discussed the possibility of effectively analyzing electronic medical records without using any manual annotations, and evaluate the performance which an approach based on the fusion of two machine-learning methods for the prediction and diagnosis of respiratory diseases. Comparison of the original diagnostic program and our proposed method is shown in Figure 1. Our proposed method provided reliable assistance without requiring any changes to the original diagnostic procedure. Despite the known association between diseases, the models could predict these diseases, and benefited a wide range of patients. In turn, we were able to identify the common features between the diseases that affected prediction. The respiratory system big data platform was used to train and test multiple models for the prediction of these diseases. 


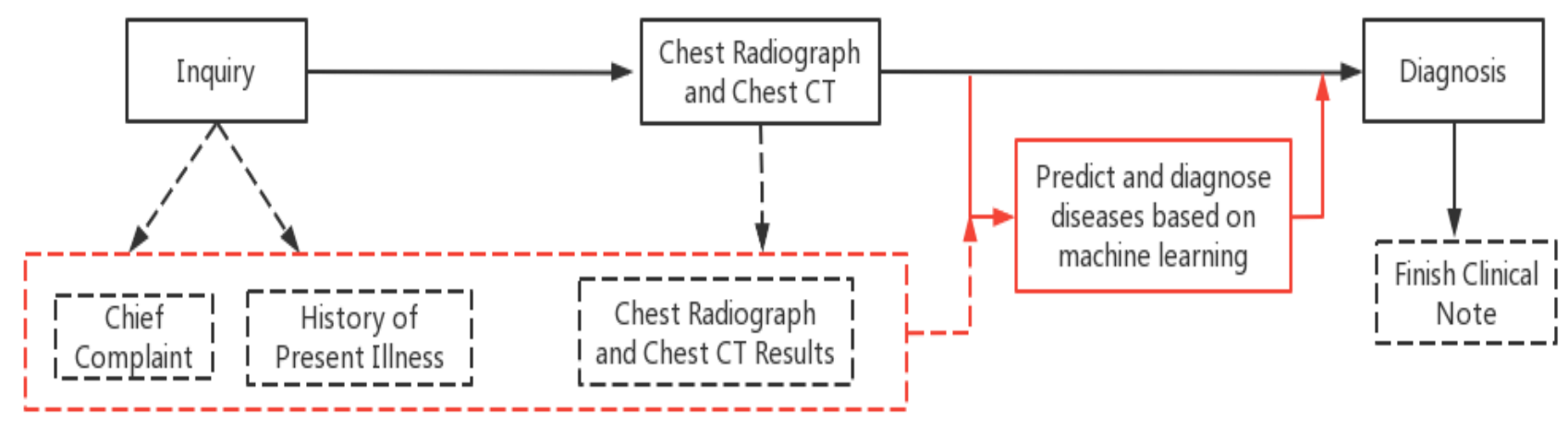

86

Figure 1. Original diagnostic procedures and our proposed method. Black solid line and boxes are

original procedure, black dashed lines and boxes are generated clinical notes at each step. Red solid lines are the additional step of using the proposed method and the red dashed box and line are the existing clinical notes used by the proposed method.

\section{Materials and Methods}

2.1 Research design We collected the medical records of inpatients in the respiratory department, including chief complaint, history of present illness, and chest computed tomography (CT). Preprocessing of clinical records with“ jieba” word segmentation module, and the BERT model was used to perform word vectorization on the text. The first stage consisted of convolutional layers and maximum pooling layers, and the second stage consisted of long-short-term memory network (LSTM) layers. The partial and total information of the fused feature set was encoded by the convolutional layers, while LSTM layers decoded the encoded information. The framework of the method based on machine learning to predict and diagnose respiratory diseases is shown in Figure 2. The development and evaluation of the solution were performed in the Python environment [13]. 
103

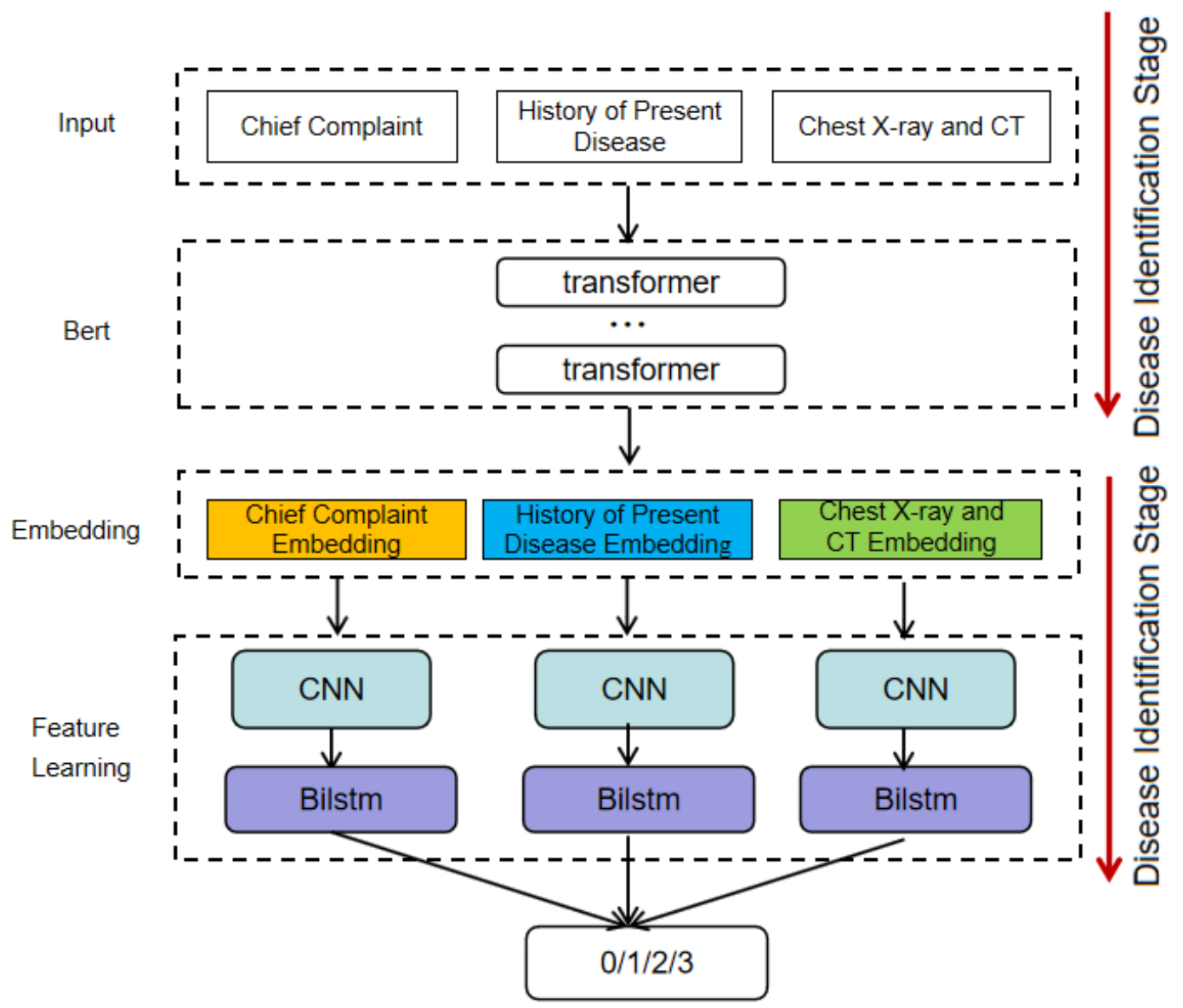

Figure 2. Overall framework of method based on machine learning to predict and diagnose

respiratory diseases.

2.2 Data requirements (1) Clinical records from January 2018 to August 2021 were collected from the Respiratory Department of the First People's Hospital of Kashi, which is a grade AAA hospital in the Southern Xinjiang . (2) All clinical records were collected for hospitalized patients diagnosed with COPD, PTB, PTE or bronchiectasis disease, excluding patients with any two or more of these diseases at the same time. (3) For patients admitted to hospital several times during this period, we collected the first admission records. (4) We collected the following information from the clinical records: age, sex, occupation, ethnicity, current medical history, chief complaint, 
imaging examination (chest X-ray and chest CT), disease history, smoking history, allergy history, and physical examination results.

2.3 Preprocessing clinical records The clinical records included chief complaints, history of present illness and CT imaging results. (1) Stop word setting: filter out adverbs, conjunctions, prepositions and modal auxiliary words in the text, and same words that have no actual meaning or have nothing to do with disease diagnosis terms. (2) Special symbol filtering: eliminate all kinds of separators and connectors in the text, such as punctuation, (“,”, “.”, “-”, etc.) and some meaningless separators (“space”, “| ”, etc.) and other symbols (“*”, “ ‘”, etc.). “?”, “+” and “ - ” indicate suspicion of disease, positive and negative, and needed to be retained. (3) Word segmentation dictionary settings: this study uses the "jieba" word segmentation module [14], and the Medical Professional Term Dictionary compiled by Tsinghua University was introduced into the module as the word segmentation dictionary, improving the efficiency of model word segmentation process. (4) To unify the phrasing of test results, all measurements were unified into the most commonly used version.

2.4 Disease identification stage The network structure of the disease identification stage is shown in the lower half of Figure 2. Instead of directly processing text input, the text data were fed into the word embedding to generate an embedding for each word. All texts obtained word vectors through the BERT model [15], and these word vectors were mapped into a high dimensional vector space $V 2 \mathrm{R} C$ using the word $2 v e c$ method. Each sentence was converted into a text embedding $\mathrm{T}=\left[\mathrm{t}_{1} ; \mathrm{t}_{2} ; \cdots ; \mathrm{t}_{\mathrm{L}}\right] 2$ 
$\mathrm{RC} \times L$, with each $t_{i} 2 \mathrm{~V}$. The main complaint, history of present illness and CT image description text $\mathrm{T}$ were passed into two convolution layers for initial feature extraction. Both convolution layers had a kernel size of three, and through these two layers, we extracted hidden information between adjacent words [16]. History of present illness and CT image description were long texts, and there was no pooling operation in these convolutional layers. Therefore, these texts was extracted contextual information through a BILSTM model [17] that composed of forward LSTM and backward LSTM.

\subsubsection{Basic models TextCNN is a variant of CNN (convolutional neural} network). TextCNN uses a k-dimensional vector to represent a word in a sentence [18]. We used the "jieba" word segmentation module to perform word segmentation according to the Dictionary of Medical Professional Terminology. Each word corresponded to a one-dimensional vector, which was entered into $\mathrm{CNN}$ for convolution. The network model consisted of 200 filters whose window sizes were 2 , 3 and 4. The specific model structure is shown in Figure 3. The training model consisted of an embedding layer, convolutional layer, pooling layer, and fully connected layer. If the eigenmaps were obtained, we pooled them according to the maximum value of each convolution value. Feature extraction was the main function of the convolution and pooling layers [19]. It extracted the main features from the text sequences of certain lengths through partial word order information and further synthesized the main features into advanced features [20]. 


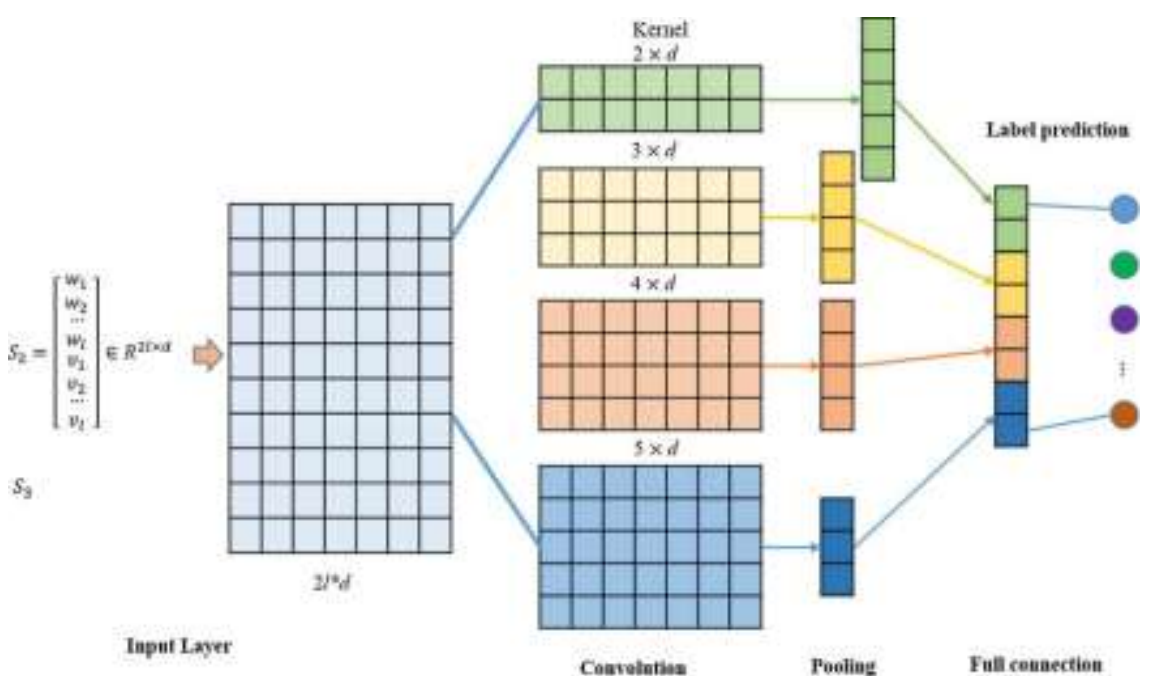

157

Figure 3. Convolutional neural network.

LSTM is a variable length neural network, and it consists of input gate $i$, forgetting gate $f$, and output gate $h[21,22]$. The equations are shown :

$$
\begin{gathered}
i_{t}=\sigma\left(W_{i} \cdot\left[h_{t-1}, x_{t}\right]+b_{i}\right) \\
f_{t}=\sigma\left(W_{f} \cdot\left[h_{t-1}, x_{t}\right]+b_{f}\right) \\
\widetilde{C_{t}}=\tanh \left(W_{c} \cdot\left[h_{t-1}, x_{t}\right]+b_{c}\right) \\
C_{t}=f_{t} * C_{t-1}+i_{t} * \widetilde{C_{t}} \\
o_{t}=\sigma\left(W_{o}\left[h_{t-1}, x_{t}\right]+b_{o}\right) \\
h_{t}=O_{t} * \tanh \left(C_{t}\right)
\end{gathered}
$$

$i_{t}$ is the input gate, while $f_{t}$ is the forget gate, and $O_{t}$ is the output gate at moment $t . \widetilde{C_{t}}$ is the input in the neuron at time $t . C_{t}$ is the updated value in the neuron at time $t . h_{t}$ stores the value of the hidden layer at time $t$ and before. The value of $\sigma$ is the activation function sigmoid. $W$ and $b$ are the weight and bias terms.

2.4.2 CNN-BILSTM model We proposed the CNN-BILSTM model, which uses a BILSTM layer and a CNN layer to extract data features. BILSTM is a bidirectional LSTM that extracts bidirectional features of text at the same time to obtain better 
174

classification results. BILSTM can capture the two-way semantic dependence from front to back and from back to front through two LSTMs in different directions, thereby effectively combining contextual information [23]. The features extracted using maximum-pooling layers are often passed to the fully connected layer for classification in CNN networks. However, in the proposed CNN network, the sequence of deep features passed to the LSTM layer rather than directly through the fully connected layer for classification. The CNN network efficiently extracted the text, while the LSTM network detected long-short-term dependencies [24]. The proposed model was introduced for prediction and diagnosis of respiratory diseases. The CNN-BILSTM model contained two phases (Figure 4). Phase one included convolution layers and maximum-pooling layers, and phase two consisted of the LSTM layer. The partial and total information of the fused feature was encoded by the convolution layers, while the LSTM layer decoded the encoded information.

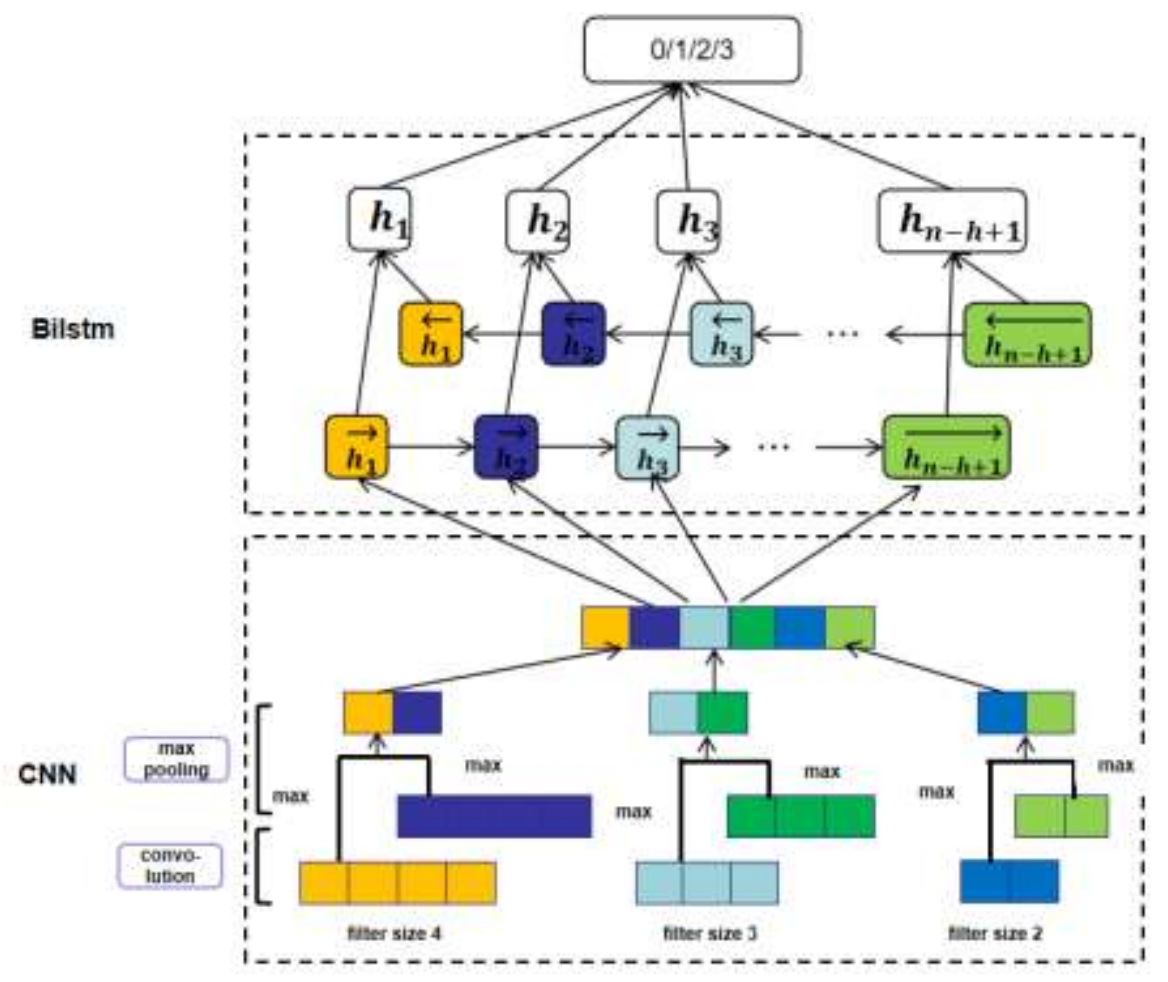


2.5 Evaluation matrices We chose three evaluation matrices such as F1 score, positive cases. If disease was defined a positive criterion, recall described the proportion of all real patients identified by the machine-learning method and treated in hospital. Precision was the ratio of all correctly classified medical records to all actually classified medical records. $P$ and $r$ were defined as follows:

$\begin{aligned} p & =\frac{\mathrm{TP}}{\mathrm{TP}+\mathrm{FP}} \\ r & =\frac{\mathrm{TP}}{\mathrm{TP}+\mathrm{FN}}\end{aligned}$

where TP, FN and FP were true positive rate, false negative rate and false positive rate. the F1 Score was a comprehensive metric that combined precision and recall. The larger the value, the better the system performance. $F 1$ score was defined as

201 follows:

$$
F 1=\frac{2 \cdot p \cdot r}{p+r}
$$

\section{Results}

3.1 Dataset We collected 3,422 eligible patients, and all clinical records were written in Mandarin. The percentages of each diseases are shown in Figure 5a. There were 1,741 patients with PTB, 400 with PTE, 686 with bronchiectasis, and 595 with COPD. 

shown in Figure 5e.

210
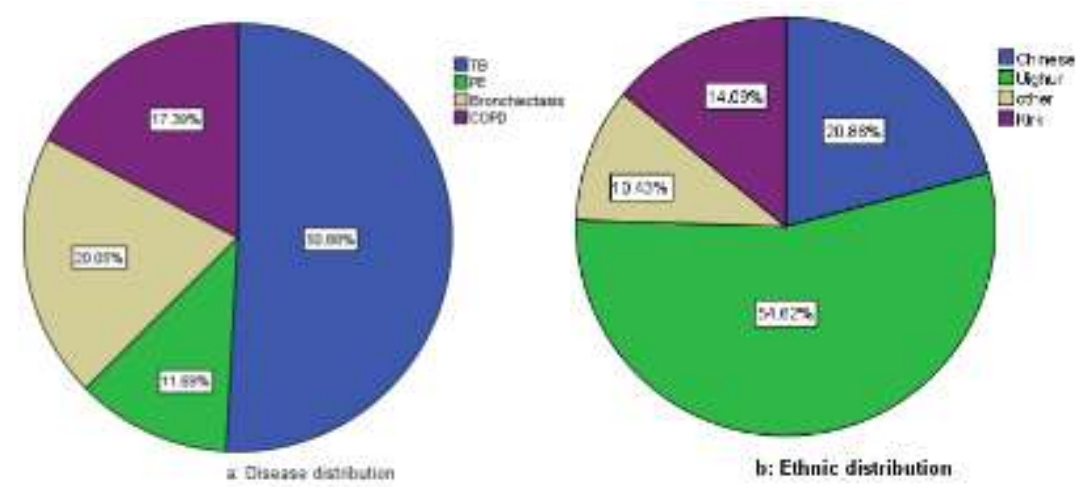

211
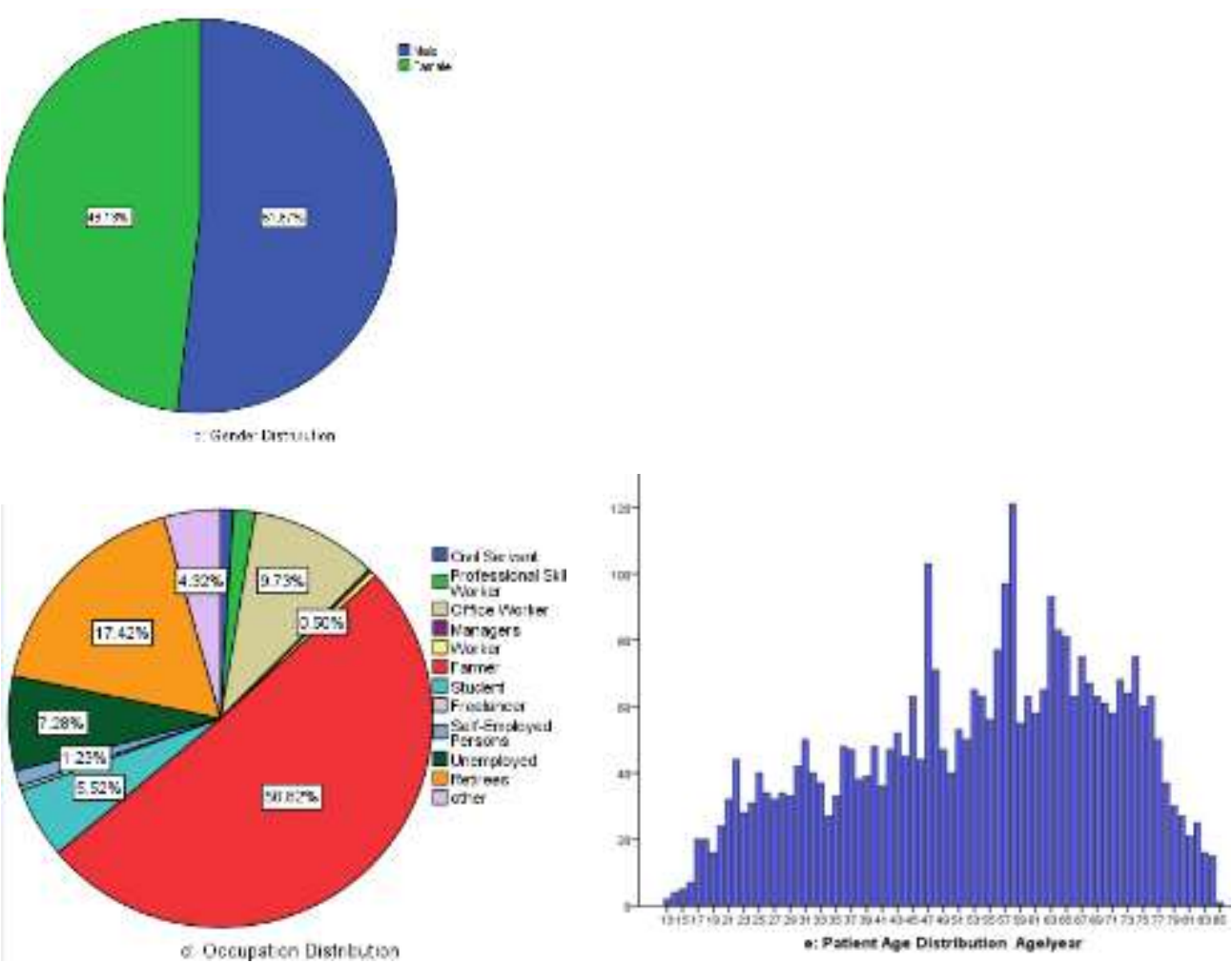

Figure 5. Dataset statistics. (a) Disease distribution, (b) ethnic distribution, (c) gender distribution,

(d) occupation distribution, (e) age distribution.

\subsection{Model comparison}

To better validate the performance of our proposed method for disease predict

217 and diagnose, we also compared our method with other machine-learning methods.

218 Deep-learning methods improved P from below 0.6 to about 0.81 , and our proposed 

(Table 1). Our proposed method had a significantly higher performance.

Table 1. Performance comparison between the proposed method and multiple benchmark

\begin{tabular}{|c|c|c|c|}
\hline method & $\mathrm{P}$ & $\mathrm{r}$ & F1 score \\
\hline Logistic Regression & 0.58 & 0.62 & 0.60 \\
\hline Decision Tree & 0.53 & 0.56 & 0.54 \\
\hline SVM & 0.68 & 0.65 & 0.66 \\
\hline $\mathrm{CNN}$ & 0.85 & 0.84 & 0.84 \\
\hline BILSTM & 0.77 & 0.81 & 0.79 \\
\hline proposed & 0.89 & 0.87 & 0.88 \\
\hline method(CNN-BILSTM) & & & \\
\hline
\end{tabular}

223

\subsection{Confusion matrix results}

To provide an in-depth understanding of the proposed method's results, we report the confusion matrix of each disease in Figure 6. FP and FN values of CNN, BILSTM and CNN-BILSTM methods were limited to a reasonable range. The FP and

FN of the proposed method were lower than those of the CNN and BILSTM methods, while TP was higher. 

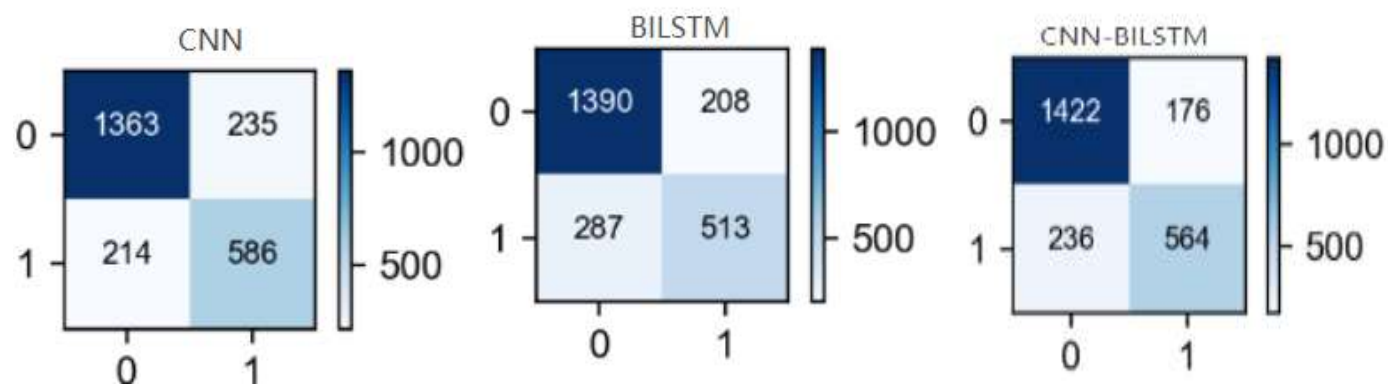

Figure 6. Confusion matrices of each method. Horizontal is predicted value, vertical is actual

value, " 0 "is negative, " 1 " is positive.

\section{Discussion}

There are many types of respiratory diseases, and the common symptoms are

obviously homogeneous. It is difficult to accurately determine the type of disease

based on medical history and physical examination, and misdiagnosis of these

diseases leads to inappropriate treatment resulting in prolonged recovery time and

for diagnosis of respiratory diseases based on artificial intelligence can achieve

diagnosis and prediction of respiratory diseases, and provide diagnostic information medical resource allocation [26].

In this study, we explored the possibility of using deep learning to analyze

244 original clinical records and identify respiratory diseases with similar symptoms,

245 including COPD, PTE, PTB and bronchiectasis. Our proposed method was

significantly better than traditional machine learning and deep learning methods, 
present illness are routine contents in clinical medical records. This information is helpful when a patient is admitted to hospital and can help doctors make an initial diagnosis, and $\mathrm{CT}$ imaging can provide a better basis for diagnosis. In the clinical records, the chief complaint is a short text with distinct features, mainly composed of patient symptom characteristics. History of present illness is a long text that mainly describes details of the patients' condition and is the result of past diagnosis and treatment. The description of chest images is mainly based on description and summary of medical terms by radiologists in combination with the patients' condition and CT results. Therefore, we collected main complaint, history of present illness, and CT imaging, and established a method for prediction and diagnosis of respiratory diseases.

Traditional machine-learning methods include Logistic Regression (LR), Decision Tree(DT), support vector machine(SVM) [27-29], and various deep learning methods recently used in analysis of clinical records, including the more advanced methods BILSTM and TextCNN. WordCNN has been proven to be a lightweight but effective architecture in multiple text analysis tasks [30-31]. Traditional machine learning methods can not effectively extract features. The core idea of TextCNN captures local features of text, extracting text information through different convolution kernels [32].Over the past few years, a large number of clinical studies have used various type of machine learning.Researchers such as M Patrício [33] used machine learning algorithms to predict breast cancer in blood sample data compared with traditional methods, and found that machine learning methods greatly shortened the diagnosis 
time and improved the accuracy. a combined deep learning and multi-level feature extraction methodology(CNN-LSTM) were proposed to identify Covid-19 CT scans and chest X-rays [34].A CNN-LSTM hybrid forecasting model has been proposed, which can precisely foresee the COVID-19 episode across India contrasted with other conventional models (i.e., LSTM and ARIMA). The proposed model uses convolutional layers to extract meaningful information and learn from a given time series dataset[35]. These studies used deep learning to achieve certain performance improvements compared with traditional machine-learning methods. In our study, the CNN-BILSTM method generally outperformed deep-learning models and traditional machine-learning methods in predicting and diagnosing diseases.

This study had the following limitations. The population was mainly in the Kashi area of Southern Xinjiang, and applicability to the wider population is limited. The epidemiology of different regions differs, so the method needs to be corrected in practical application. Primary healthcare units cannot fully cover the required inspections. Our dataset consisted of approximately 3,400 clinical records, and further increasing the data set size could improve the performance of our method. Numerical data such as BMI and blood pressure are sparse, and some fields have $90 \%$ empty entries, so we excluded numerical data in this study. Extracting numerical values from clinical records more accurately and generating denser data should improve the performance.

\section{Acknowledgements}


The authors thank to the First Peoples Hospital of Kashi for their vigorous cooperation, and the participants for whom these studies were created and who generously volunteer time in completing the tasks. We thank International Science Editing ( http://www.internationalscienceediting.com ) for editing this manuscript.

\section{Funding}

This work was supported by The State Key Laboratory of Pathogenesis, Prevention and Treatment of High Incidence Diseases in Central

Asia,(SKL-HIDCA-2020-KS1) .State Key Laboratory of Pathogenesis, Prevention and Treatment of High Incidence Diseases in Central Asia.(SKL-HIDCA-2020-10)

Tianshan Innovation Team Plan Of Autonomous Region (2020D14013).

\section{Availability of data and materials}

The datasets used and analysed during this study can be available online at xjkshospital.com/grxjb.

\section{Author's contribution}

Li Li, Alimu Ayiguli and Qiyun Luan designed the study, implemented the model and drafted the manuscript . Xiaoguang Zou and Jiangtao Ren participated in data preprocessing and manuscript revision, experiment design. Boyi Yang,Xuemei Zhong,Hui Gong, Abudureherman Zulipikaer,Yilamujiang Subinuer,Jingran Xu performed experiments and analyses. All authors have read and approved the final version of this manuscript.

\section{Ethics approval and consent to participate}


312

We confirmed that this study's all methods were carried out in accordance with relevant guidelines and regulations, and all experimental protocols were approved by First People's Hospital of Kashi.Meanwhile, we confirmed that informed consent was obtained from all subjects and/or their legal guardian(s).

\section{Consent for publication}

Not applicable.

\section{Competing interests}

The authors declare that they have no competing interests.

\section{Declarations}

We confirmed that this study's all methods were carried out in accordance with relevant guidelines and regulations, and all experimental protocols were approved by First People's Hospital of Kashi.Meanwhile, we confirmed that informed consent was obtained from all subjects and/or their legal guardian(s).

\section{References}

1.Bagdonas E, Raudoniute J, Bruzauskaite I, Aldonyte R.Int J Chron Obstruct. Novel aspects of pathogenesis and regeneration mechanismsin COPD,Pulmon Dis. 2015 2;10:995-1013.

2. Cardona PJ.Pathogenesis of tuberculosis and other mycobacteriosis.

Enferm Infecc Microbiol Clin (Engl Ed),2018;36(1):38-46.

3. Chang AB, Bush A, Grimwood K.Bronchiectasis in children: diagnosis and treatment,Lancet. 2018;392(10150):866-879.

4. Mishina T, Miyajima M, Watanabe A.Management of Pulmonary Thromboembolism,Kyobu Geka. 2017;70(8):678-682. 
334

5. Morris PE, Berry MJ, Files DC, Thompson JC.Standardized Rehabilitation and Hospital Length of Stay Among Patients With Acute Respiratory Failure: A Randomized Clinical Trial.JAMA,2016;315(24):2694-702.

6. Gong $\mathrm{H}$, Ren J, Xu J, et al. SMAD3 rs36221701 T $>$ C polymorphism impacts COPD susceptibility in the Kashi population[J]. Gene, 2021, 808: 145970.

7.Peiffer-S N, Rawson TM, Ahmad R, et al. Machine learning for clinical decision support in infectious diseases: a narrative review of current applications.Clin Microbiol Infect. 2020;26(5):584-595.

8.Xi Y, Tian C L, Qian L. A study of deep learning methods for de-identification of clinical notes in cross-institute settings.BMC Med Inform Decis Mak. 2019; 19;5: 232.

9.Gans D, Kralewski J, Hammons T, Dowd B. Medical groups' adoption of electronic health records and information systems. Health Aff. 2005;24(5):1323-33.

10.Raghupathi W, Raghupathi V. Big data analytics in healthcare: promise and potential. Health Inf Sci Syst. 2014;2(1):3.

11. Gang Yu, Zhongzhi Y, Yemin Shi. Identifification of Pediatric Respiratory Diseases Using Fine-grained Diagnosis System.J Biomed Inform. 2021;117:103754.doi: 10.1016/j.jbi.

12.Deo RC. Machine Learning in Medicine[J].Circulation,2015,132(20):1920-30.

13. Pedregosa F, et al. Scikit-learn: Machine learning in Python. J Mach Learn Res. 2011;12:2825-2830.

14.Shuai cao.New Word Detection Algorithm Combining Correlation Confidence and Jieba Word Segmentation.Computer Systems \& Applications,2020,29(5):144-151 
355

356

357

358

359

360

361

362

363

364

365

366

367

368

369

370

371

372

373

374

375

15.KANTARDZIC M. Data Mining: Concepts, Models, Methods and Algorithms[M]. New Jersey:

John Wiley and Sons, 2011

16.Dashdorj Z, Song M. An application of convolutional neural networks with salient features

for relation classification.BMC Bioinformatics. 2019 ,29;20:244-250.

17. B. Shickel, P. J. Tighe, A. Bihorac,et al. Deep ehr: a survey of recent advances in deep learning techniques for electronic health record analysis.IEEE J Biomed Health

Inform.2018,22(5):1589-1604.

18.Iqbal H. S.Machine Learning: Algorithms, Real-World Applications and Research

Directions.SN Comput Sci. 2021; 2(3): 160-168.

19.Stephen W, Kirk R, Surabhi D,et al.Deep learning in clinical natural language processing: a methodical review.J Am Med Inform Assoc. 2020, 27(3): 457-470.

20.Guergana K. S, Ioana D, Folami A,et al.Use of Natural Language Processing to Extract

Clinical Cancer Phenotypes from Electronic Medical Records.Cancer Res, 2019,79(21): 54635470.

21.Qiu-JL, Hsin-YC, Wei-BZ,et al.A Multi-Task Group Bi-LSTM Networks Application on Electrocardiogram Classification.IEEE J Transl Eng Health Med. 2020;

8: doi: 10.1109/JTEHM.2019.2952610.

22.Nazanin F, Kary F.A Novel LSTM for Multivariate Time Series with Massive

Missingness.Sensors (Basel) 2020 May; 20(10): 2832-36.

23.Wen XL, Hai DR, Chang YL,et al.A Method Based on GA-CNN-LSTM for Daily Tourist Flow Prediction at Scenic Spots.Entropy (Basel) ,2020; 22(3): 261. 
376

377

378

379

380

381

382

383

384

385

386

387

388

389

390

391

392

393

394

395

396

397

24.Ning C, Yue C, Wan qG ,et al.An Improved Deep Learning Model:S-TextBLCNN for

Traditional Chinese Medicine Formula Classification.Front Genet. 2021; 12:

807825.doi: 10.3389/fgene.2021.807825.

25.Lee J, Yoon W, Kim S, et al. BioBERT: a pre-trained biomedical language representation model for biomedical text mining.Bioinformatics. 2020,36(4):1234-1240.

26.Abdelkader NB, Sofia O, Abderrahmane L,et al.End-to-End AI-Based Point-of-Care

Diagnosis System for Classifying Respiratory Illnesses and Early Detection of COVID-19: A

Theoretical Framework,Front Med (Lausanne) 2021; 8: 585578.

27.Tolles J, Meurer WJ.Logistic Regression: Relating Patient Characteristics to

Outcomes.JAMA,2016,316(5):533-4.

28.Calamuneri A, Donato L, Scimone C,et al. On machine learning in biomedicine. Life Safety and Security. 2017;5(12):96-99.

29. Erickson BJ, Korfiatis P, Akkus Z,et al. Machine learning for medical imaging. RadioGraphics. 2017;37(2):505-515.

30.Mobadersany P, Yousefi S, Amgad M, et al. Predicting cancer outcomes from histology and genomics using convolutional networks. Proc. Natl. Acad. Sci. USA. 2018;115:E2970-E2979. doi: 10.1073/pnas.1717139115.

31.Wei S, Chao G, Yue Z,et al.A Time Series Data Filling Method Based on LSTM-Taking the Stem Moisture as an Example.Sensors (Basel) ,2020; 20(18): 5045.

32.J. Wrenn, I. Jones, K. Lanaghan, etal. Estimating Patient's Length of Stay in the Emergency Department with an Artificial Neural Network. AMIA Symposium, 2005,1155-1155.

PMCID: PMC1560706 

presence of breast cancer[J]. Bmc Cancer, 2018, 18(1):181-188.

400 34.Hamad N, Ali A, Bin S.A CNN-LSTM network with multi-level feature extraction-based

401 approach for automated detection of coronavirus from CT scan and X-ray images.Appl Soft

402 Comput. 2021; 113: 107918.

403 35.Shwet K, Pramod KM.India perspective: CNN-LSTM hybrid deep learning model-based

404 COVID-19 prediction and current status of medical resource availability.Soft

405 comput. $2021 ; 10 ; 19: 1-20$. 